\title{
Modelización de la formación de recubrimientos de WC-Co por proyección HVOF sobre sustratos de cobre ${ }^{(\bullet)}$
}

\author{
V.V. Sobolev ${ }^{(*)}$, J.M. Guilemany ${ }^{(*)}$ y J.A. Calero ${ }^{(*)}$ \\ Resumen En el presente trabajo se ha investigado la simulación matemática de la transferencia de calor entre un \\ recubrimiento de WC-Co y un sustrato de cobre durante la proyección HVOF. Este modelo incluye el \\ estudio de la variación de termperatura, solidificación del recubrimiento, la fusión y posterior solidifi- \\ cación en la región interfacial del sustrato, y caracerísticas especiales de la interacción térmica sustra- \\ to-recubrimiento. Los resultados obtenidos han sido utilizados en la modelización del desarrollo de la \\ estructura del recubrimiento y la adherencia durante la proyección HVOF de polvos de WC-Co en un \\ sustrato de cobre. Se han considerado dos tipos de sustrato: sustrato con superficies pulida y rugosa. \\ Se han estudiado las variaciones de los tiempos de solidificación, de la velocidad de solidificación, del \\ gradiente térmico, y de la velocidad de enfriamiento en el recubrimiento y en la región interfacial del \\ sustrato. Se discute el desarrollo de las estructuras amorfas y cristalinas en el recubrimiento y de la \\ estructura cristalina en la zona interfacial del sustrato. Asimismo, se estudia el comportamiento del \\ tamaño de los cristales y de la distancia intercristalina con respecto a los parámetros de proyección y a \\ la morfología de la superficie del sustrato. Se han determinado las condiciones óptimas para la forma- \\ ción de estructuras de grano fino y denso. Se han considerado también los cambios estructurales en el \\ sustrato en estado sólido debidos al calentamiento y enfriamiento rápidos. Asimismo, se han investiga- \\ do los mecanismos térmicos y mecánicos de la adherencia sustrato-recubrimiento. Por último, decir \\ que los resultados teóricos concuerdan con los resultados experimentales obtenidos.
}

Palabras clave: Proyección HVOF. Recubrimientos de WC-Co. Sustrato de cobre. Transferencia de calor. Estructura.

\section{Modelling of the formation of WC-Co coatings by HVOF spraying onto copper substrates}

\begin{abstract}
Present paper deals with the mathematical simulation of the heat transfer between a WC-Co coating and a copper substrate during HVOF spraying. This modelling includes the investigation of temperature variation, coating solidification, melting and subsequent solidification in the substrate interfacial region and specific features of the substrate-coating thermal interaction. The results obtained are used for modelling of the development of the coating structure and adhesion during HVOF spraying of the WC-Co powder on a copper substrate. Two types of substrate were considered: smooth (polished) and rough. Variations of solidification times, solidification velocity, thermal gradient and cooling velocity in the coating and substrate interfacial region are studied. Development of the amorphous and crystalline structures in the coating and of the crystalline structure in the substrate interfacial region is discussed. Behaviour of the crystal size and intercrystalline distance with respect to the thermal spray parameters and morphology of the substrate surface is analyzed. Optimal conditions for the formation of fine and dense crystalline structure are determined. Structural changes in the solid state of the substrate occuring because of heating and rapid cooling are considered. Mechanical and thermal mechanisms of development of the substrate-coating adhesion are discussed. Results obtained agree well with experimental data.
\end{abstract}

Keywords: HVOF spraying. WC-Co coating. Copper substrate. Heat transfer. Structure.

(•) Trabajo recibido el día 12 de noviembre de 1996.

(*) Dpto. de Ciencia de Materiales e Ingeniería Metalúrgica. Centro de Proyección Térmica. Dpto. de Ingeniería Química y Metalurgia. Universidad de Barcelona. C/ Martí i Franquès, 1. 08028-Barcelona (España).

\section{INTRODUCCIÓN}

El desarrollo de la estructura del recubrimiento, y con ello de las propiedades, depende esencialmente de la transferencia de calor entre el sustrato y 
el recubrimiento, que tiene lugar durante la depositación y solidificación del mismo (1-3). Particularmente, se refiere al calor transferido en la región interfacial del sustrato con el recubrimiento, la cual incluye la primera capa y la zona interfacial del sustrato, debido a que en esta región se produce una unión de tipo adhesivo entre el recubrimiento y el sustrato (4-6).

Para un determinado tipo de recubrimiento, la dinámica de la transferencia de calor depende significativamente del material que forma el sustrato y de la morfología de la superficie del sustrato. Se investigó el calor transferido entre el recubrimiento de WC-Co y el sustrato con superfice pulida de acero y entre este recubrimiento y un sustrato con superficie pulida de una aleación de aluminio (2, 3 y 7-9). Se han establecido diferencias muy marcadas entre la cinética de solidificación y fusión del recubrimiento y la de solidificación de la región interfacial del sustrato debido a las propiedades termofísicas diferentes de los materiales que constituyen el sustrato.

La morfología de la superfície del sustrato desempeña también un papel importante en la interacción térmica entre el sustrato y el recubrimiento. Esto se debe, principalmente, a la influencia en la rugosidad de la superficie en la deformación de la partícula proyectada durante la proyección térmica (9 y 10). Con una superficie de sustrato rugosa, el grosor de la lámina del recubrimiento proyectado aumenta y su longitud disminuye en comparación con lo que sucede para una superficie de sustrato pulida (9). La solidificación de la lámina también influye en el proceso de la formación de la propia lámina a partir de las partículas proyectadas (11).

El flujo térmico de la lámina delgada formada en la superficie pulida del sustrato, se dispersa rápidamente hacia el interior del mismo; por ello, el grosor de la capa fundida en la región interfacial del sustrato resulta ser pequeño. En el caso de superficie de sustrato más rugosa, la lámina formada es más gruesa y la temperatura en el sustrato es mayor que para el caso anterior; por lo que la profundidad de la zona fundida será mayor.

La rugosidad en la superficie del sustrato también contribuye al aumento de la resistencia de contacto térmico en la interfase sustrato-recubrimiento. Esto conduce a la disminución de la transferencia de calor entre el recubrimiento y el sustrato y, por tanto, al aumento de la temperatura en el sustrato y al aumento de la profundidad de la zona fundida en la región interfacial del mismo.

\section{DESCRIPCION DEL MODELO}

El modelo desarrollado involucra cálculos de la distribución de temperaturas en el sustrato, $T_{1}$, y en el recubrimiento, $T_{2}$, las cuales pueden ser consideradas como funciones de tan sólo las coordenadas transversal, $r$, y del tiempo, $t$. Esto es correcto para la región central de la muestra proyectada; sin embargo, el efecto de borde puede influir en la periferia del recubrimiento. Debido a esto, los cálculos ignoran el efecto de borde y tan sólo tienen en cuenta la parte central del recubrimiento.

En la superfice superior de la capa de recubrimiento se aplica una condición límite que incluye el intercambio de calor entre los gases y el propio splat mediante el coeficiente de transferencia de calor, $\alpha$. En la interfase entre el sustrato y el recubrimiento, la resistencia térmica de contacto, $R_{\mathrm{C}}$, se tiene en cuenta a través de un coeficiente de transferencia de calor, $\alpha_{C}$, que es inversamente proporcional a la resistencia térmica de contacto, $R_{\mathrm{C}}$, la cual aumenta con el aumento de la rugosidad superficial del sustrato. Una descripción detallada del modelo se presenta en (2, 9 y 10$)$.

El principal objetivo del estudio estaba dirigido a la interacción térmica entre la primera capa del recubrimiento y el sustrato. También se ha estudiado la influencia de las siguientes capas de recubrimiento sobre la transferencia de calor (12).

Las temperaturas calculadas se han empleado para la determinación de los parámetros estructurales. En la versión básica de los cálculos se han introducido los siguientes parámetros: grosor de sustrato de $3 \mathrm{~mm}$; espesor de capa de recubrimiento, $\delta=15 \mu \mathrm{m}$; temperatura inicial del sustrato, $T_{10}$ $=20{ }^{\circ} \mathrm{C}$; coeficiente de transferencia de calor en la superficie superior de la capa de recubrimiento, $\alpha=$ $1.000 \mathrm{Wm}^{-2} \mathrm{~K}^{-1}$; coeficiente de contacto térmico para una superficie pulida, $\alpha_{C}=6,67 \cdot 10^{6} \mathrm{Wm}^{-2} \mathrm{~K}^{-1}$, y para una superficie rugosa (chorreada), $\alpha_{C}=$ $3,33 \cdot 10^{6} \mathrm{Wm}^{-2} \mathrm{~K}^{-1}$; la temperatura del gas en la superficie superior de la capa de recubrimiento es de $500{ }^{\circ} \mathrm{C}$.

\section{RESULTADOS Y DISCUSION}

\subsection{Proceso de transferencia de calor}

\subsubsection{Primera capa de recubrimiento}

La primera capa de recubrimiento se forma por el impacto y la deformación y posterior solidificación de las gotas fundidas proyectadas sobre la superficie del sustrato. La interacción en el impacto entre la gota y el recubrimiento y la formación del splat se describe en (6 y 9). Para el objetivo del presente análisis se ha tenido en cuenta la diferencia en la morfología de la superficie del sustrato mediante diferentes coeficientes de contacto térmico.

La fase líquida en la capa de recubrimiento solidifica al transferir el calor del recubrimiento al 
sustrato. Un posterior enfriamiento de la capa de recubrimiento ocurre como resultado de la pérdida de calor desde la zona superior del recubrimiento con el medio ambiente. Pero este enfriamiento es esencialmente menor que el debido al sustrato. Sin embargo, dada la competencia entre estos dos tipos de procesos, los perfiles de temperatura a través de la capa de recubrimiento presentarán un valor máximo, el cual estará próximo a la superficie libre del recubrimiento.

La modelización de la solidificación en la primera capa del recubrimiento indica que para la superficie chorreada o rugosa el tiempo de solidificación, $t_{\mathrm{S} 2}$, varía de forma no uniforme con el aumento de la temperatura inicial del sustrato, $T_{10}$, y de la capa de recubrimiento, $T_{20}$ (Fig. 1). Este comportamiento se debe al hecho de que un aumento en $T_{10}$ y $T_{20}$, por una parte, conduce al aumento en el contenido calorífico del sustrato y del recubrimiento, respectivamente, y por lo tanto contribuye a una mayor duración del proceso de solidificación de la primera capa del recubrimiento. Pero, por otra parte, un aumento en $T_{10}$ y $T_{20}$ provoca un aumento en los gradientes de temperatura entre la capa de recubrimiento y el sustrato y esto causa una aceleración del proceso de solidificación de la primera capa. La competencia entre ambas tendencias conduce al comportamiento no uniforme de $t_{\mathrm{S} 2}$ frente a $T_{10}$ y $T_{20}$. La disminución de la resistencia de con-

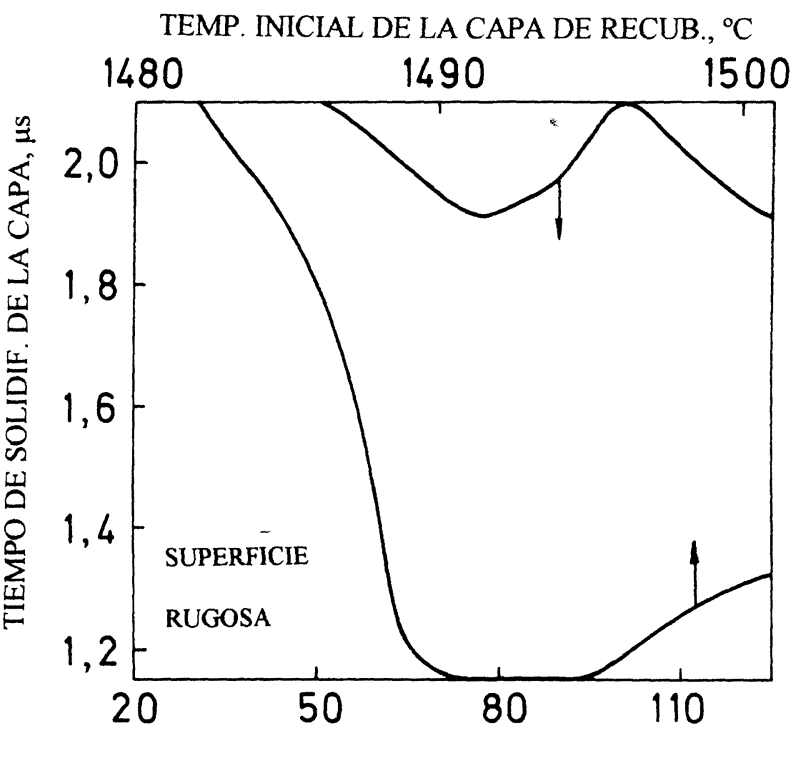

TEMP. INICIAL DEL SUSTRATO, ${ }^{\circ} \mathrm{C}$

FIG. 1.- Variación del tiempo de solidificación de la capa de recubrimiento respecto a la temperatura inicial de sustrato y de la capa de recubrimiento, para superficie rugosa del sustrato.

FIG. 1.- Variation of solidification time of coating layer with respect to initial temperatures of substrate and layer for rough substrate surface. tacto térmico en el caso de la superficie pulida provoca que el comportamiento de $t_{\mathrm{S} 2}$ con respecto a la temperatura inicial del sustrato sea más uniforme (Fig. 2). La figura 3 muestra que el tiempo de solidificación $t_{\mathrm{S} 2}$ aumenta con el aumento del espesor de capa.

La cinética del proceso de solidificación para la primera capa se presenta en la tabla I. El intervalo de tiempo desde el impacto de las partículas con la formación de la capa y el inicio de la solidificación en la interfase sustrato-recubrimiento, viene dado por el tiempo $t_{\mathrm{L} 1}$. La isoterma de liquidus se mueve posteriormente hacia la superficie libre de la capa, a la que llega tras un intervalo de tiempo, $t_{\mathrm{L} 2}$. La total solidificación en la interfase sustrato-capa ocurre después de un intervalo de tiempo, $t_{\mathrm{S} 1}$, y la primera capa queda totalmente sólida tras un intervalo de tiempo $t_{\mathrm{S} 2}$. Los valores de $t_{\mathrm{L} 1}$ y $t_{\mathrm{S} 1}$ son idénticos e iguales, $0,019 \mu \mathrm{s}$ para el sustrato con superficie pulida, y de $0,191 \mu$ s para el sustrato con superficie rugosa. Este aumento en $t_{\mathrm{L} 1}$ y $t_{\mathrm{S} 1}$ se debe a la disminución del calor transferido desde la capa hacia el sustrato cuando el coeficiente térmico de contacto aumenta.

El parámetro $\tau_{1}$, definido como $\left(t_{\mathrm{L} 2}-t_{\mathrm{L} 1}\right) t_{\mathrm{S} 2}{ }^{-1}$ siempre es menor que $\tau_{2}$, definido como $\left(t_{\mathrm{S} 2}-t_{\mathrm{S} 1}\right)$ $t_{\mathrm{S} 2}{ }^{-1}$. Esto implica que la isoterma de liquidus se mueve más rápida que la de solidus. La diferencia entre $\tau_{1}$ y $\tau_{2}$ disminuye cuando lo hace la resistencia térmica de contacto. Como resultado, el líquido

TEMP. INICIAL DE LA CAPA DE RECUB., ${ }^{\circ} \mathrm{C}$

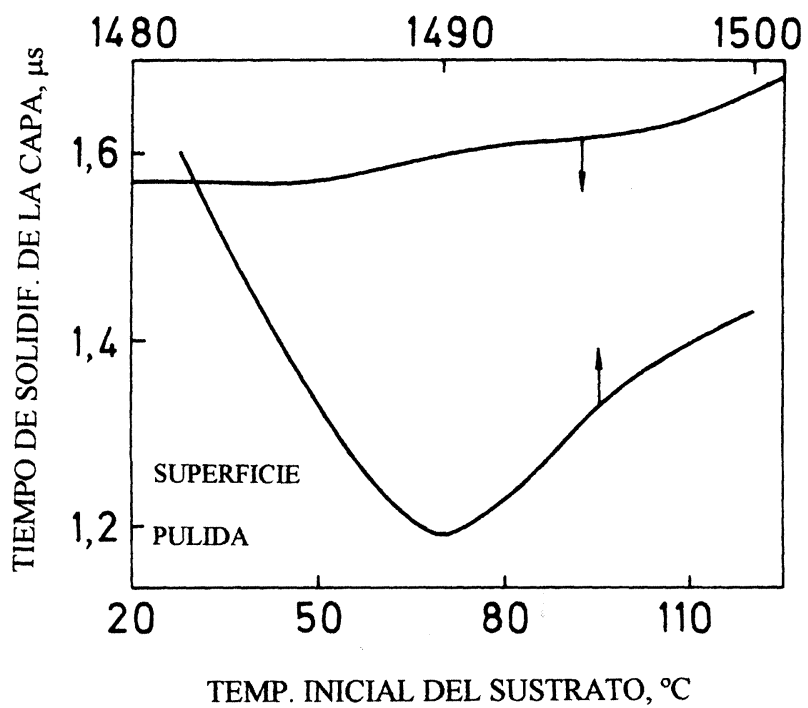

FIG. 2.- Variación del tiempo de solidificación de la capa de recubrimiento respecto a la temperatura inicial del sustrato y de la capa de recubrimiento, para superficie pulida de sustrato.

FIG. 2.-Variation of solidification time of coating layer with respect to initial temperatures of substrate and layer for smooth substrate surface. 


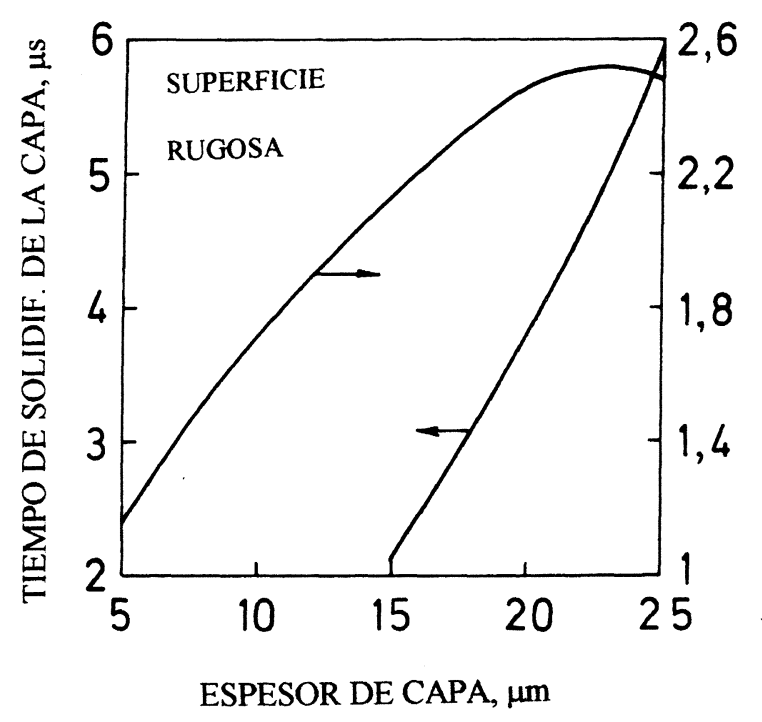

FIG. 3.- Variación del tiempo de solidificación de la capa de recubrimiento y sustrato respecto al grosor de capa para superficie rugosa de sustrato.

FIG. 3.- Variation of solidification time of coating layer and substrate with respect to layer thickness for rough substrate surface.

está presente en la solidificación de la capa para un sustrato pulido un 10-80 \% del tiempo total requerido para la solidificación, $t_{\mathrm{S} 2}$, mientras que para un sustrato rugoso está un 40-70\%. La capa solidificada permanece en un estado sólido-líquido (mushy) cerca de la superficie un 85-97\% del tiempo total de solidificación en el caso de trabajar con un sustrato chorreado, y casi un $100 \%$ para un sustrato pulido.

Estos resultados difieren de los obtenidos para recubrimientos de WC-Co depositados sobre sustratos de acero o aleaciones de aluminio, debido a las diferencias en las propiedades termofísicas de los materiales que forman el sustrato. En el caso del sustrato de acero, en la solididficación de la primera capa de recubrimiento, la fase líquida permanece un 20-30\% del tiempo total de solidificación, y la zona de dos fases sólida-líquido o zona mushy permanece en la parte superior de la primera capa de recubrimiento entre un 60 y un $80 \%$ (2). Para el sustrato de aleación de aluminio, la fase líquida en la primera capa de recubrimiento permanece durante un $60-80 \%$ de $t_{\mathrm{S} 2}$, mientras que la zona de mushy existe durante un 20 a un $40 \%$ (7).

De los valores de $\tau_{2}$ se puede deducir que la fase líquida y sólida están presentes durante un período insignificante del proceso de solidificación de la primera capa de recubrimiento. Esto es especialmente cierto en la proximidad de la superficie libre de la capa antes de la finalización del proceso de solidificación, lo que aumenta las condiciones favorables para el desarrollo de la porosidad en el recubrimiento $(6,13-15)$. Los resultados muestran que el valor de $\tau_{2}$ aumenta con el aumento de $T_{10}, T_{20} \mathrm{y}$ $\delta$ para un sustrato pulido, pero $\tau_{2}$ disminuye con el aumento de $T_{10}$ y $T_{20}$ para un sustrato rugoso.

\subsubsection{Región interfacial del sustrato}

La historia térmica del sustrato implica los siguientes estadios: (i) calentamiento como resultado del calor transferido desde la primera capa del recubrimiento; (ii) fusión de la zona interfacial del sustrato si se alcanza una temperatura suficientemente elevada; (iii) posterior solidificación y enfriamiento del sustrato; (iv) recalentamiento y enfriamiento del sustrato debido a la llegada de la segunda y subsiguientes capas de recubrimiento.

En el presente caso se ha utilizado un cobre como sustrato con la suficiente pureza como para fundir a $1.083{ }^{\circ} \mathrm{C}$. La fusión tiene lugar cuando se supera la temperatura de solidus, $T_{\mathrm{S} 1}$, y esto ocurre en un tiempo, $t_{\mathrm{S} 1}$. La isoterma de solidus se mueve desde la interfase sustrato-recubrimiento hacia el interior del sustrato una distancia, $h_{\mathrm{S}}$, mientras que continúa el calentamiento del mismo, alcanza su valor de penetración máximo, $h_{\mathrm{Sm}}$, cuando la temperatura en el sustrato es inferior a $T_{\mathrm{S} 1}$, lo que ocurre en un tiempo, $t_{\mathrm{S} 3}$.

TABLA I.- Variación de tiempos característicos de solidificación de la primera capa de recubrimiento respecto a parámetros de proyección y morfología de la superficie del sustrato

TABLE I.- Variation of characteristic times of solidification of the first coating layer with respect to parameters of spraying and morphology of substrate surface

\begin{tabular}{|c|c|c|c|c|c|c|c|c|c|c|}
\hline \multicolumn{2}{|c|}{} & \multicolumn{4}{|c|}{ Superficie pulida } & \multicolumn{4}{c|}{ Superficie rugosa } \\
\hline$T_{10},{ }^{\circ} \mathrm{C}$ & $T_{20},{ }^{\circ} \mathrm{C}$ & $\delta, \mu \mathrm{m}$ & $t_{\mathrm{L} 2}, \mu \mathrm{s}$ & $t_{\mathrm{s} 2}, \mu \mathrm{s}$ & $\tau_{1}$ & $\tau_{2}$ & $t_{\mathrm{L} 2}, \mu \mathrm{s}$ & $t_{\mathrm{S} 2}, \mu \mathrm{s}$ & $\tau_{1}$ & $\tau_{2}$ \\
\hline 20 & 1.482 & 15 & 1,22 & 1,57 & 0,77 & 0,988 & 1,15 & 2,11 & 0,46 & 0,91 \\
125 & 1.482 & 15 & 1,30 & 1,68 & 0,76 & 0,989 & 0,96 & 1,91 & 0,40 & 0,90 \\
20 & 1.500 & 15 & 1,19 & 1,44 & 0,81 & 0,997 & 1,15 & 1,34 & 0,72 & 0,86 \\
20 & 1.482 & 25 & 3,43 & 4,31 & 0,79 & 0,996 & 4,03 & 5,93 & 0,65 & 0,97 \\
\hline
\end{tabular}


Durante el calentamiento, la temperatura de liquidus se alcanza en la interfase en un tiempo, $t_{\mathrm{L} 1}$, de tal forma que se tiene una zona de profundidad, $h_{\mathrm{L}}$, totalmente en estado líquido. La profundidad máxima de la isoterma líquidus, $h_{\mathrm{Lm}}$, se alcanza en un tiempo $t_{\mathrm{L} 3}$. Los resultados de simulación muestran que $t_{\mathrm{L} 1}=t_{\mathrm{S} 1}=0,0383 \mu \mathrm{s}$ para una superficie pulida del sustrato y $t_{\mathrm{L} 1}=t_{\mathrm{S} 1}=0,191 \mu \mathrm{s}$ para una superficie rugosa. Durante la proyección HVOF, la temperatura alcanzada puede no ser suficiente como para fundir totalmente la región interfacial del sustrato, por lo que en la región interfacial permanece en estado de dos fases, o mushy zone (2 y $3)$. En el caso que interesa, esta es la situación que tiene lugar, y el valor de $h_{\mathrm{Sm}}$ encontrado para la versión básica de los cálculos es de aproximadamente $0,1 \mu \mathrm{m}$ para superficie pulida y $0,5 \mu \mathrm{m}$ para superficie rugosa.

Cuando el calor se elimina, la solidificación en la región interfacial del sustrato comienza y la isoterma de liquidus se mueve hacia la interfase sustrato-recubrimiento, la cual se alcanza en un tiempo $t_{\mathrm{L} 4}$. En un tiempo $t_{\mathrm{S} 4}$, la isoterma solidus alcanza la interfase y la solidificación se completa en su totalidad. Es por ello por lo que se considera $t_{\mathrm{S} 4} \mathrm{el}$ tiempo en el que se completa la solidificación de la zona interfacial del sustrato, cuyo valor es principalmente el tiempo de solidificación del movimiento de la isoterma. Los parámetros $\tau_{3}=\left(t_{\mathrm{L} 4}-t_{\mathrm{L} 3}\right) t_{\mathrm{S} 4}{ }^{-1}$ y $\tau_{4}=$ $\left(t_{\mathrm{S} 4}-t_{\mathrm{S} 3}\right) t_{\mathrm{S} 4}{ }^{-1}$ son los tiempos relativos de movimiento de las isotermas solidus y liquidus, respectivamente.

Para el caso del sustrato con superficie pulida, los valores de $t_{\mathrm{L} 3}$ y $t_{\mathrm{S} 3}$ varían entre 0,1 y $0,2 \mu \mathrm{s}$ cuando la temperatura inicial de la capa varía entre 20 y $125{ }^{\circ} \mathrm{C}$, la temperatura inicial del sustrato se encuentra entre 1.482 y $1.500{ }^{\circ} \mathrm{C}$ y la variación del espesor de capa está entre 15 y $25 \mu \mathrm{m}$. En el caso del sustrato con rugosidad superficial, bajo estas variaciones de $T_{10}, T_{20} \mathrm{y} \delta$, los valores de $t_{\mathrm{L} 3}$ y $t_{\mathrm{S} 3}$ son iguales a $0,765 \mu \mathrm{s}$.

Lo más representativo son las variaciones de $t_{\mathrm{S} 4}$ y $\tau_{4}$ que se muestran en la tabla II. La zona interfa- cial del sustrato permanece en estado de dos fases durante un significativo período de su tiempo de solidificación y este período, para el caso de la superficie chorreada, es mayor que para el caso de superficie pulida.

Los valores de $h_{\mathrm{S}}$ aumentan con el tiempo, alcanzan un valor máximo, $h_{\mathrm{Sm}}, \mathrm{y}$, posteriormente, disminuyen. Las variaciones de $h_{\mathrm{Sm}}$ respecto a $T_{10}$ son más importantes que en relación con $T_{20}$, puesto que la temperatura inicial del sustrato tiene más influencia en la transferencia de calor en la región interfacial del sustrato que la temperatura inicial de la capa. Los valores de $h_{\text {Sm }}$ para la superficie rugosa son mayores que para la pulida, debido a la intensa acumulación de calor que tiene lugar en el primer caso.

El tiempo de solidificación, $t_{\mathrm{S} 4}$, de la región interfacial del sustrato para el caso de superficie rugosa es casi el doble que para la solidificación de la primera capa de recubrimiento. En el caso de superficie pulida, el tiempo de solidificación de la región interfacial del sustrato es cerca de un orden de magnitud' superior al de la solidificación de la primera capa de recubrimiento. Esto difiere sustancialmente de la situación cuando un recubrimiento de WC-Co se proyecta sobre un sustrato de acero. En este caso, el valor de $t_{\mathrm{S} 4}$ es cerca de dos órdenes de magnitud superior al $t_{\mathrm{S} 2}$ debido a la diferencia en las propiedades termofísicas del cobre y del acero.

\subsubsection{Siguientes capas de recubrimiento}

De acuerdo con el sistema de rotación y parámetros utilizados en la proyección HVOF (pistola CDS PT100 en el Centro de Proyección Térmica de la Universidad de Barcelona), la segunda y siguientes capas de recubrimiento se depositan con un intervalo de $1,32 \mathrm{~s}$.

$\mathrm{La}$ influencia de la transferencia de calor de las siguientes capas de recubrimiento depende significativamente de las propiedades termofísicas del material del sustrato y del recubrimiento. Tanto los

TABLA II.- Variación de tiempos característicos de solidificación de la zona interfacial del sustrato respecto a los parámetros de proyección y a la morfología de la superficie del sustrato

TABLE II.- Variation of characteristic times of solidification of the substrate interfacial region with respect to parameters of spraying and morphology of substrate surface

\begin{tabular}{|c|c|c|c|c|c|c|}
\hline \multicolumn{2}{|c|}{} & \multicolumn{2}{c|}{ Superficie pulida } & \multicolumn{2}{c|}{ Superficie rugosa } \\
\hline$T_{10},{ }^{\circ} \mathrm{C}$ & $T_{20},{ }^{\circ} \mathrm{C}$ & $\delta, \mu \mathrm{m}$ & $t_{\mathrm{s} 4}, \mu \mathrm{s}$ & $\tau_{4}$ & $t_{\mathrm{s} 4}, \mu \mathrm{s}$ & $\tau_{4}$ \\
\hline 20 & 1.482 & 15 & 0,23 & 0,500 & 2,68 & 0,714 \\
125 & 1.482 & 15 & 1,76 & 0,881 & 4,11 & 0,814 \\
20 & 1.500 & 15 & 0,40 & 0,667 & 2,87 & 0,733 \\
20 & 1.482 & 25 & 0,23 & 0,500 & 2,49 & 0,692 \\
\hline
\end{tabular}


resultados de simulación como los resultados experimentales de caracterización del recubrimiento muestran que para los recubrimientos de WC-Co proyectados por HVOF sobre un sustrato de acero, la influencia de una segunda capa es pequeña y con las sucesivas capas esta influencia decrece, siendo casi irrelevante en la cuarta capa ( 2 y 3 ). Es por ello que, en este caso, la transferencia de calor en la interfase está determinada principalmente por la interacción térmica entre el sustrato y la primera capa de recubrimiento.

La situación es diferente cuando el recubrimiento de WC-Co se obtiene sobre un sustrato de aleación de aluminio $(\mathrm{Al}-4 \% \mathrm{Cu})(7$ y 8$)$. Debido a las propiedades termofísicas de esta aleación, las capas posteriores depositadas influyen de forma importante en la transferencia de calor y el desarrollo de la estructura. El calor transferido desde la segunda capa provoca la refusión de la región interfacial del sustrato y su resolidificación.

La situación difiere de igual modo cuando se proyecta WC-Co sobre un sustrato de cobre. Los resultados de la simulación muestran que durante el enfriamiento de la segunda capa, la temperatura en la interfase sustrato-recubrimiento aumenta hasta $\operatorname{los} 480{ }^{\circ} \mathrm{C}$, que no es suficiente como para provocar la refusión de la región interfacial del sustrato. La temperatura máxima, $T_{\mathrm{m}}$ en la interfase disminuye con el aumento del número de capas depositadas (Fig. 4). Esta temperatura depende ligeramente de la morfología de la superficie del sustrato. En la figura 4 se presentan los valores de $T_{\mathrm{m}}$ para el caso de superficie pulida del sustrato.

En la figura 5 se presenta la variación de la temperatura máxima en la interfase entre la primera y la segunda capa $\left(T_{\mathrm{m}}{ }^{(1,2)}\right)$, entre la segunda y la tercera $\left(T_{\mathrm{m}}{ }^{(2,3)}\right)$, entre la tercera y la cuarta $\left(T_{\mathrm{m}}{ }^{(3,4)}\right)$ y

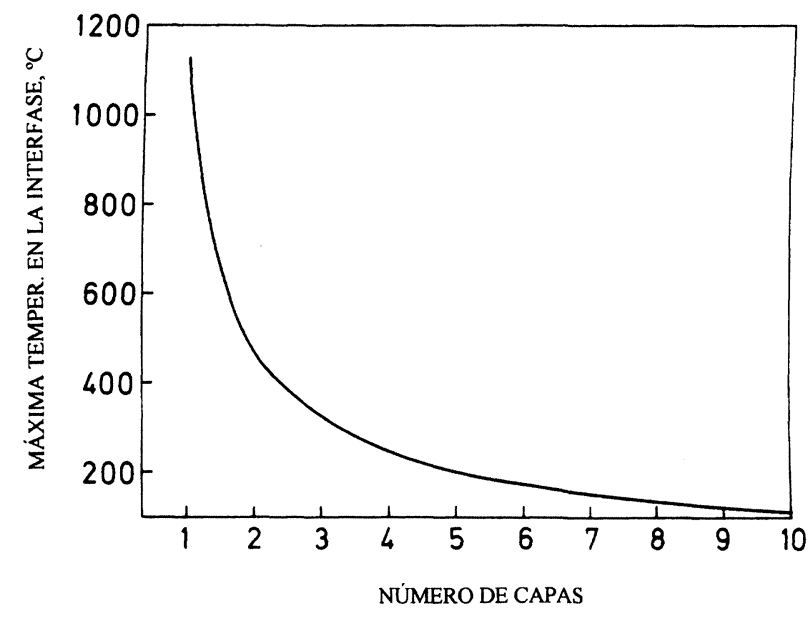

FIG. 4.- Variación de la temperatura máxima interfacial respecto al número de capas.

FIG. 4.- Variation of maximum interfacial temperature with respect to layer number.

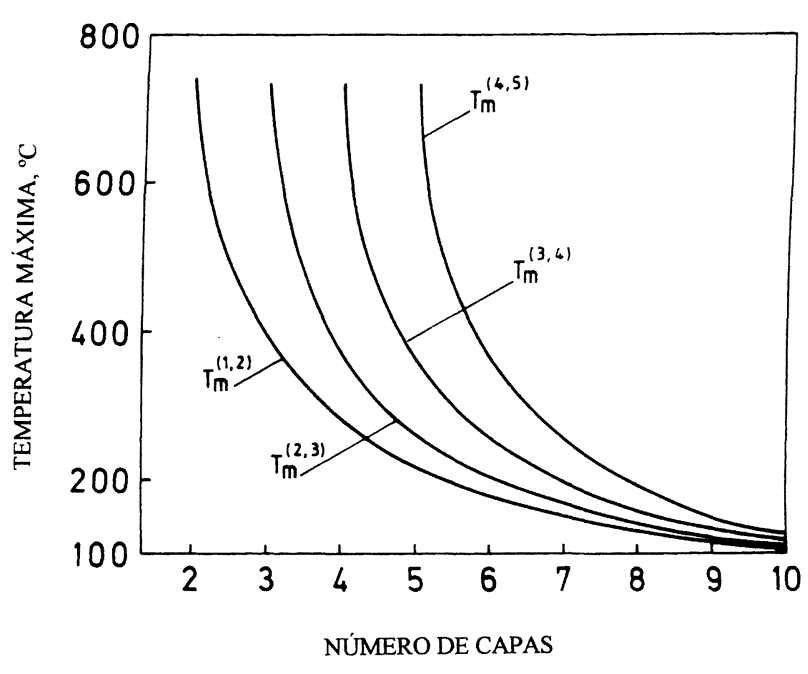

FIG. 5.- Variación de la temperatura máxima en las interfases entre diferentes capas de recubrimiento con respecto al número de capas.

FIG. 5.- Variation of maximum temperature at interfaces between different coating layers with respect to layer number.

entre la cuarta y la quinta $\left(T_{\mathrm{m}}{ }^{(4,5)}\right)$ respecto al número de capas. Estas temperaturas disminuyen con el aumento del número de capa y prácticamente no dependen de la morfología de la superficie del sustrato.

\subsection{Formación de la estructura}

\subsubsection{Primera capa de recubrimiento}

En la primera capa de recubrimiento y en sucesivas capas, tanto la estructura amorfa como la cristalina pueden formarse debido a la elevada velocidad de enfriamiento. Primeramente, se demostró que la fase metálica del recubrimiento de WC-Co proyectada sobre un sustrato de acero con un contenido bajo de carbono tenía una elevada facilidad para la formación de estructuras vítreas y, por ello, una alta probabilidad de formación de estructuras amorfas (2). Esta probabilidad fue confirmada mediante la caracterización del recubrimiento (16 y 17). La estructura del recubrimiento de WC-Co sobre un sustrato de aleación de aluminio y sobre un sustrato de cobre son similares a la de un sustrato de acero (18 y 19).

Debido al calor transferido desde las partículas fundidas en su impacto, las capas de recubrimiento previamente depositadas pueden ser recalentadas y cuando la temperatura alcanza 0,4 a 0,6 de la temperatura liquidus de la fase metálica que forma el recubrimiento, la estructura amorfa de este puede ser parcialmente destruida y transformada en estructura cristalina (20). Estas temperaturas 
pueden alcanzarse durante la proyección térmica (16 y 17).

La estructura de grano fino de la capa de recubrimiento se caracteriza por el parámetro $\xi$, definido como el tamaño de cristal, y el parámetro $\eta$, definido como la distancia entre cristales. Los valores de $\xi$ y $\eta$ pueden determinarse usando las velocidades de solidificación y enfriamiento calculadas y los valores de los gradientes térmicos de las isotermas solidus y liquidus. Se utilizan las siguientes fórmulas para el cálculo de $\xi$ y de $\eta(21)$ :

$$
\begin{aligned}
& \xi=a v_{\mathrm{S} 2}^{\mathrm{b}} G_{\mathrm{S} 2}^{\mathrm{c}} \\
& \eta=d v_{\mathrm{CS} 2}^{e}
\end{aligned}
$$

Para los cálculos de la estructura de la capa de recubrimiento, se utilizan los siguientes parámetros en las ecuaciones [1] y [2]: $a=10^{-4}, b=-0,25$, $c=-0,5, d=3,8$ y $e=-0,4$.

El valor medio de $\xi$ para el caso de superficie pulida del sustrato varía de forma no uniforme con el aumento de $T_{10}$ y dismuye con el aumento de $T_{20}$ (Fig. 6). La figura 7 muestra que, en el caso de la superficie de sustrato rugosa, el valor medio de $\xi$ presenta un comportamiento no uniforme con respecto a $T_{10}$ y $T_{20}$. El valor medio de $\eta$ varía no uniformemente con el aumento de $T_{10}$ y $T_{20}$ para el caso de superficie de sustrato rugosa. Un aumento

TEMP. INICIAL DE LA CAPA DE RECUB., ${ }^{\circ} \mathrm{C}$

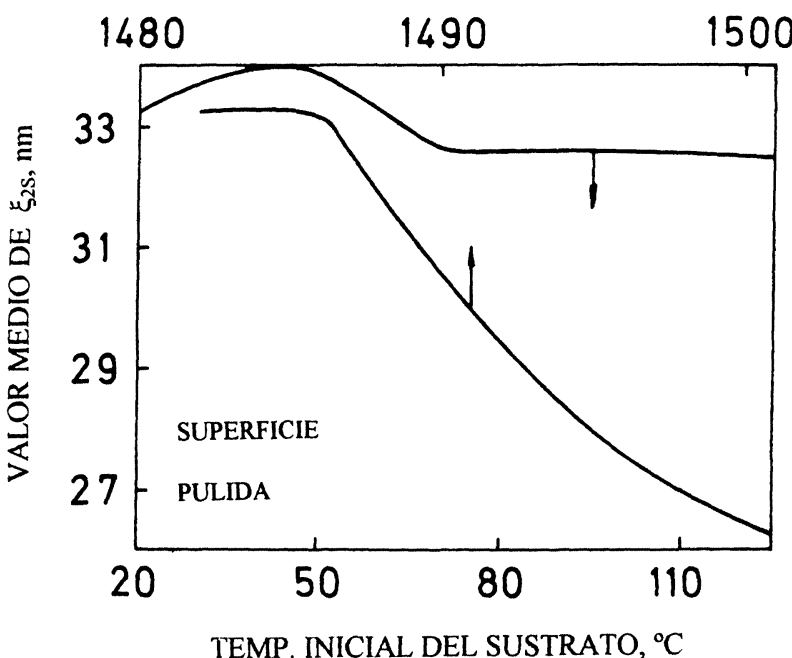

FIG. 6.- Variación del valor medio del tamaño cristalino, $\xi_{\mathrm{S} 2}$, respecto a la temperatura inicial del sustrato y de la capa de recubrimiento para superficie pulida.

FIG. 6.- Variation of mean value of crystal size $\xi_{\mathrm{S} 2}$ with respect to initial temperatures of substrate and coating layer for smooth surface.
TEMP. INICIAL DE LA CAPA DE RECUB., ${ }^{\circ} \mathrm{C}$

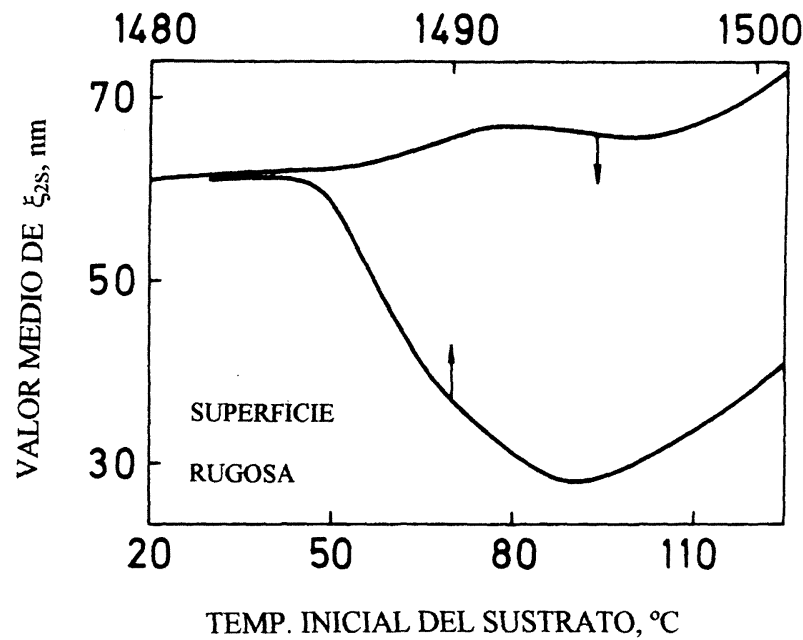

FIG. 7.- Variación del valor medio del tamaño cristalino, $\xi_{\mathrm{S} 2}$, respecto a la temperatura inicial del sustrato y de la capa de recubrimiento para superfi cie rugosa.

FIG. 7.- Variation of mean value of crystal size $\xi_{\mathrm{S} 2}$ with respect to initial temperatures of substrate and coating layer for rough surface.

en el espesor de capa provoca un aumento en los valores medios de $\xi$ y de $\eta$.

El valor mínimo de $\xi$, correspondiente a la estructura más fina para el caso de superficie pulida de sustrato, debe verificarse cuando $T_{10}=70-120$ ${ }^{\circ} \mathrm{C}, T_{20}=1.495-1.500{ }^{\circ} \mathrm{C}$ y $\delta=5-10 \mu \mathrm{m}$. Para el caso de superfice rugosa, la estructura de cristal fino se obtendría cuando $T_{10}=20-50{ }^{\circ} \mathrm{C}$ y $T_{20}=1$ $495^{\circ} \mathrm{C}$.

La estructura cristalina más densa tendrá lugar cuando $\eta$ sea mínimo, y esto ocurre en el caso de superficie de sustrato pulida cuando $T_{10}=120^{\circ} \mathrm{C}$, $T_{20}=1.495-1.500{ }^{\circ} \mathrm{C} \mathrm{y} \delta=5-10 \mu \mathrm{m}$, y en el caso de superficie rugosa cuando $T_{10}=20-50^{\circ} \mathrm{C}$ y $T_{20}=$ $1.495^{\circ} \mathrm{C}$.

\subsubsection{Desarrollo de la estructura cristalina en la región interfacial del sustrato}

El tamaño de cristal $\xi$, en la región interfacial del sustrato viene dado por la ecuación [1] y la distancia intercristalina $\eta$ por la ecuación [21]:

$$
\eta=f \cdot G^{-g}
$$

Los parámetros utilizados han sido: $a=3 \cdot 10^{-4}$, $b=-0,25$ y $\mathrm{c}=-0,5$ en [1], y $f=0,88$ y $g=0,275$ en [3].

Los resultados de simulación indican que el tamaño de cristal, $\xi$, disminuye en la dirección del 
interior del sustrato. La distancia intercristalina, $\eta$, también disminuye en la misma dirección.

Para el caso de la superficie pulida del sustrato, el valor medio de $\xi$ y de $\eta$ crece con la temperatura inicial de la capa y del sustrato, puesto que tiene lugar una disminución en las velocidades de solidificación y de enfriamiento y en los gradientes térmicos (Fig. 8). En este caso, el tamaño de cristal medio y la distancia intercristalina prácticamente no varían con el aumento del espesor de la capa.

En el caso de superficie rugosa del sustrato, los valores medios de $\xi$ y de $\eta$ varían de forma no uniforme con respecto a $T_{10}$ y $\delta$. Estos parámetros muestran también un comportamiento no uniforme respecto a $T_{20}$ alcanzando el valor mínimo a $T_{20}=$ $1.490{ }^{\circ} \mathrm{C}$.

Los valores mínimos de $\xi$ y de $\eta$ corresponden a la estructura cristalina más fina y más densa, respectivamente, en la región interfacial del sustrato, que en el caso de sustrato pulido tiene lugar cuando $T_{10}=20-50{ }^{\circ} \mathrm{C}$ y $T_{20}=1.482-1.485{ }^{\circ} \mathrm{C}$, y para el caso de sustrato rugoso estos valores mínimos se obtienen cuando $T_{10}=90-100{ }^{\circ} \mathrm{C}, T_{20}=1.490{ }^{\circ} \mathrm{C}$ y $\delta=5-10 \mu \mathrm{m}$.

\subsubsection{Transformaciones estructurales en el estado sólido}

En los materiales utilizados como sustrato para aplicaciones de proyección térmica, pueden tener lugar ciertos cambios estructurales en estado sólido a causa del calentamiento y enfriamiento rápidos.

TEMP. INICIAL DE LA CAPA DE RECUB., ${ }^{\circ} \mathrm{C}$

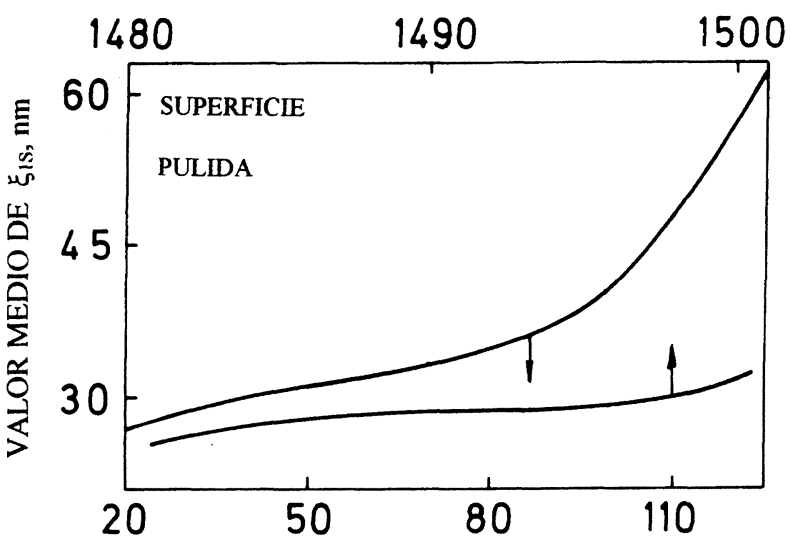

TEMP. INICIAL DEL SUSTRATO,${ }^{\circ} \mathrm{C}$

FIG. 8.- Variación del valor medio del tamaño cristalino, $\xi_{\mathrm{S} 1}$, respecto a la temperatura inicial del sustrato y de la capa de recubrimiento para superfi cie pulida.

FIG. 8.- Variation of mean value of crystal size $\xi_{\mathrm{S} 1}$ with respect to initial temperatures of substrate and coating layer for smooth surface.
Tales cambios han sido caracterizados en sustratos de acero y de aleaciones de aluminio recubiertos de WC-Co por proyección HVOF (16-18).

En el caso de sustrato de cobre, el chorreado proporcionó una zona de deformación mucho mayor en profundidad que la obtenida para el caso del acero y del aluminio. Estas diferencias pueden ser causadas por el endurecimiento por deformación característico de los materiales. Los pulsos térmicos desde los splats iniciales son suficientes para que tenga lugar una parcial recristalización en el sustrato de cobre. Pero la relación temperaturatiempo característica de la transferencia de calor es insuficiente para eliminar las estructuras relativamente estables en bandas de deformación desarrolladas, debido a la energía transferida por el impacto de las partículas que producen el chorreado. Como resultado, se tiene, cerca de la interfase entre el sustrato y el recubrimiento, características estructurales similares a las obtenidas cuando se funde y solidifica rápidamente el cobre. Los resultados de simulación muestran que la temperatura máxima de la interfase de 5 a $10 \mu \mathrm{m}$ es de $925-825^{\circ} \mathrm{C}$, que es bastante inferior al punto de fusión del cobre.

El calor transferido desde los splats iniciales produce recristalización hasta una profundidad de aproximadamente $40 \mu \mathrm{m}$, el límite de la región deformada plásticamente. Los resultados de simulación indican que a esta profundidad desde la interfase, la temperatura máxima se encuentra entre los 550 y los $600{ }^{\circ} \mathrm{C}$ que es suficiente para producir los efectos arriba descritos.

En el caso de superficie de sustrato pulida, el calor transferido desde los splats iniciales fue suficiente como para fundir el cobre en las cercanias de la interfase, pero insuficiente como para desarrollar una zona fundida sobre toda la región interfacial. El sustrato pulido se encontró libre de cualquier trabajo en frio, por lo que la recristalización del cobre no se esperaba que tuviera lugar bajo la zona fundida. La energía térmica de los splats iniciales no fue suficiente como para producir un aumento del tamaño de grano o causar cualquier otro cambio estructural en el sustrato.

Los resultados de simulación muestran que, tanto para el sustrato pulido como para el rugoso, el calor transferido desde la segunda y sucesivas capas de recubrimiento no produce ningún cambio en el material del sustrato.

\subsubsection{Capas sucesivas del recubrimiento}

En el proceso de depositación de las sucesivas capas de recubrimiento, la distancia entre ellas y el sustrato aumenta y la solidificación de estas capas tiene lugar a velocidades menores que para la 
primera capa, por lo que los parámetros cristalino $\xi$ y $\eta$ aumentan. Estos parámetros varian con $T_{10}, T_{20}$, $\delta$ y con la morfología de la superficie del sustrato de igual manera que para el caso de la primera capa de recubrimiento.

\subsection{Adherencia entre el recubrimiento y el sustrato}

La adherencia de los recubrimientos proyectados térmicamente está fuertemente influiada por mecanismos e interacciones térmicas entre el recubrimiento y el sustrato, así como por las microestructuras desarrolladas en la región interfacial. Por esta razón se han realizado grandes esfuerzos dirigidos al análisis de estos fenómenos (3, 5, 6 y 16-19).

Para obtener una buena adherencia es necesario poner en contacto de la mejor manera posible, o sea un contacto muy íntimo, la superficie superior del sustrato y la inferior de la primera capa de recubrimiento. Durante la deformación de las partículas en el impacto sobre la superficie del sustrato se producen unas elevadas presiones (6). La presión, $P$, generada bajo el impacto en la parte central del splat, debido a que la energía cinética de la partícula es máxima, es $10^{3}-10^{4}$ veces la presión atmosférica (6). El valor de $P$ disminuye al alejarse de la parte central y dirigirse hacia la periferia. Un aumento de la energía cinética de las partículas o gotas causa un aumento en la presión, $P$, y produce un gradiente de presión brusco a lo largo del splat.

Habitualmente, la superficie del sustrato es rugosa e incluso si se considera pulida siempre existe una pequeña rugosidad. Para obtener un buen contacto entre el sustrato y el recubrimiento la presión desarrollada, $P$, debe exceder la presión capilar, $P_{\sigma}$, la cual aumenta en la interfase sustratorecubrimiento a causa de la rugosidad. Debido a esto, la relación $\zeta=P / P_{\sigma}$ puede correlacionarse con la microadherencia sustrato-recubrimiento. Los resultados obtenidos en (6) muestran que la relación (y por lo tanto la microadherencia) aumenta con un aumento en la densidad y velocidad del impacto de las partículas y la rugosidad de la superficie del sustrato. El valor de $\xi$ disminuye con el aumento de la masa perdida de la partícula debido al impacto y la tensión superficial de la interfase sustrato-partícula. La relación $\xi$ es máxima en la parte central del splat y disminuye en dirección a su periferia. Estas tendencias se corresponden con las encontradas experimentalmente para la microadherencia del sustrato-recubrimiento durante la proyección térmica (22).

Los resultados de la interacción térmica entre el sustrato y el recubrimiento obtenidos en $(2,4$ y 7$)$ permiten establecer un mecanismo adicional de for- mación de la microadherencia sustrato-recubrimiento.

La fusión parcial o completa en la zona interfacial del sustrato contribuye al desarrollo de un contacto íntimo entre el recubrimiento y el sustrato, dependiendo este contacto, esencialmente, de la morfología de la superficie del sustrato. La rugosidad superficial impide el flujo de las partículas deformadas sobre la superficie del sustrato (9-11). Aparece una elevada y localizada fuente de calor desde el splat para fundir el sustrato, lo cual es particularmente importante para la periferia del splat donde la presión, $P$, no es suficientemente alta como para desarrollar un buen enlace entre el recubrimiento y el sustrato. La zona interfacial del sustrato fundido puede enlazar realmente con el sustrato. En los experimentos realizados, la región fundida tiene una profundidad de $0,5 \mu \mathrm{m}$ y se obtiene una buena adherencia.

Con la superficie pulida, el splat se extiende sobre una gran área y, por lo tanto, el flujo térmico desde aquí hacia el sustrato es menor, y la máxima profundidad de fusión en la región interfacial es de $0,1 \mu \mathrm{m}$. Ello implica un menor tiempo para el desarrollo de un buen enlace y, por lo tanto, se obtiene una peor adherencia.

No obstante, son necesarios más trabajos para un mejor entendimiento del desarrollo de la adherencia sustrato-recubrimiento, particularmente para conseguir resultados cuantitativos de los parámetros de los procesos mecánicos y térmicos que conducen a la formación de buenas adherencias entre el material del recubrimiento y el del sustrato.

\section{COMPARACIÓN CON LOS RESULTADOS EXPERIMENTALES}

La proyección HVOF se realizó con un sistema AG PT100 de Plasma Technik, en el Centro de Proyección Térmica de la Universidad de Barcelona. Se proyectó un polvo comercial sobre un sustrato de cobre.

La microestructura del recubrimiento es similar a la descrita en (17). La microestructura de la región interfacial del sustrato depende esencialmente de la morfología de la superficie del sustrato (19) y difiere de forma importante de la obtenida con el mismo recubrimiento proyectado sobre sustratos de acero y aleación de aluminio (16-18).

\subsection{Región interfacial sustrato-recubrimiento para el caso de superficie de sustrato rugosa}

En el caso de superficie de sustrato rugosa se pueden identificar cuatro zonas en la región interfacial del sustrato. La zona 1 está en una región 
inmediatamente adyacente a la interfase y consiste en finos cristales con tamaños de $50 \mathrm{~nm}$ que son un poco mayores que los obtenidos para superficie de sustrato pulida. La profundidad de esta zona es de aproximadamente $0,5 \mu \mathrm{m}$ (19). La zona 1 es la región donde el cobre ha fundido. Esto último es coherente con los resultados de simulación que muestran que la profundidad de esta zona es de aproximadamente $0,5 \mu \mathrm{m}$ y el tamaño de los cristales de entre 40 y $60 \mathrm{~nm}$ (19).

Inmediatamente debajo de la zona 1 se observa una región de recristalización del cobre, zona 2, y una región de finos cristales (19). La primera región tiene un tamaño de grano entre 0,7 y 4-5 $\mu \mathrm{m}$ y se extiende desde $1-5 \mu \mathrm{m}$ de profundidad, pero la medida usual está cerca de 1,5-2 $\mu \mathrm{m}$. La región cristalina final tiene un tamaño de cerca de $0,5 \mu \mathrm{m}$ y el tamaño de cristal se sitúa entre 50-100 $\mathrm{nm}$. La secuencia estructural granos recristalizados-granos finos se repite de 3 a 4 veces para dar una profundidad total entre 5-8 $\mu \mathrm{m}$. La estructura observada en la zona 2 es coherente con los resultados de la simulación. Estos resultados indican que a una profundidad de entre 5-10 $\mu \mathrm{m}$, bajo la interfase entre el recubrimiento y el sustrato rugoso, se tiene una temperatura, dentro del rango de 925-700 ${ }^{\circ} \mathrm{C}$, que se mantiene por un período de cerca de $40 \mu \mathrm{s}$, lo cual parece suficiente para producir la recristalización parcial observada y la zona deformada en frio.

La zona 3 se encuentra bajo la zona 2 y consiste en cobre recristalizado, extendiéndose hasta una profundidad de entre 30 y $40 \mu \mathrm{s}$ desde la interfase. Los estudios de simulación muestran que a esta profundidad las temperaturas máximas alcanzadas están en torno a los $550-600{ }^{\circ} \mathrm{C}$ lo cual es suficiente como para observar los efectos producidos.

La zona 4 de la microestructura es idéntica a la estructura del sustrato original, como se confirma por los resultados de la historia térmica obtenidos mediante la simulación. Por todo ello, se puede afirmar que los resultados teóricos concuerdan con los obtenidos experimentalmente.

\section{CONCLUSIONES}

- La fase líquida como única fase se presenta en la solidificación de la primera capa en un 70-80 \% del tiempo de solidificación total, para la superficie pulida del sustrato, y un $40-70 \%$ del tiempo para el caso de superficie rugosa del sustrato. El estado mushy permanece en el límite superior de la capa durante un 85-97\% del tiempo de solidificación total. Esto difiere significativamente de la situación cuando el recubrimiento de WC-Co se obtiene sobre sustratos de acero o aleación de aluminio.
- Los resultados obtenidos muestran que durante el calentamiento desde la capa de recubrimiento, la región interfacial del sustrato funde parcialmente formándose una zona de dos fases sólidolíquido, o estado mushy. Para el sustrato con superfice pulida, la profundidad de la fusión es de aproximadamente $0,1 \mu \mathrm{m}$ y para la superficie rugosa $0,5 \mu \mathrm{m}$.

- La región interfacial del sustrato permanece en estado mushy durante un importante período de tiempo de solidificación, siendo mayor para el caso de superficie rugosa que para el de la superficie pulida del sustrato.

- El tiempo de solidificación total de la región interfacial del sustrato para el caso de superficie rugosa es cerca de dos veces mayor que para la primera capa de recubrimiento. Este tiempo en el caso de superficie de sustrato pulida es cerca de un orden de magnitud mayor que para la primera capa de recubrimiento.

- Durante el enfriamiento de la segunda capa del 'recubrimiento, la temperatura en la región interfacial sustrato-recubrimiento aumenta, pero este aumento no es suficiente para la refusión de la región interfacial del sustrato. La temperatura máxima en la interfase disminuye con un aumento del número de capas. Esta temperatura depende débilmente de la morfología de la superficie del sustrato. La temperatura máxima en la interfase entre diferentes capas de recubrimiento disminuye con el aumento del número de capas y prácticamente no depende de la morfología de la superficie del sustrato.

- En la primera capa de recubrimiento y en las siguientes se han encontrado, tanto estructuras amorfas como estructuras nanocristalinas, debido a las elevadas velocidades de enfriamiento. Los valores medios del tamaño de cristal, $\xi$, para superficie de sustrato suave varía de forma no uniforme con el aumento de $T_{10}$, y decrecen con el aumento de $T_{20}$. En el caso de superficie rugosa el valor medio de $\xi$ presenta un comportamiento no uniforme respecto a $T_{10}$ y $T_{20}$. El valor medio de la distancia intercristalina, $\eta$, varía de forma no uniforme con el aumento de $T_{10}$ y $T_{20}$, tanto para la superficie pulida como para la rugosa. Un aumento en el espesor de la capa de recubrimiento provoca un aumento en los valores medios de $\xi$ y de $\eta$.

- La estructura de cristal más fino en el caso de superficie de sustrato pulida puede esperarse cuando $T_{10}=70-120{ }^{\circ} \mathrm{C}, T_{20}=1.495-1.500{ }^{\circ} \mathrm{C} \mathrm{y}$ $\delta=5-10 \mu \mathrm{m}$. En el caso de superficie de sustrato rugosa, la estructura de cristal fino es probable que tenga lugar cuando $T_{10}=20-50{ }^{\circ} \mathrm{C}$ y $T_{20}$ $=1.495{ }^{\circ} \mathrm{C}$. La estructura cristalina más densa 
para superficie de sustrato pulida es probable que ocurra cuando $T_{10}=120{ }^{\circ} \mathrm{C}, T_{20}=1.495$ $1.500{ }^{\circ} \mathrm{C}$ y $\delta=5-10 \mu \mathrm{m}$.

- Durante la formación del sustrato se producen transformaciones estructurales en el estado sólido del sustrato debido al calentamiento y enfriamiento rápidos. Cerca de la interfase sustratorecubrimiento en el caso de superficie rugosa, las características estructurales son similares a las acontecidas cuando tiene lugar una fusión y una rápida solidificación. El calor transferido desde la primera capa de recubrimiento provoca una recirstalización hasta una profundidad de 40 $\mu \mathrm{m}$ para el caso de superficie de sustrato rugosa. En el caso de superficie pulida de cobre, la recristalización no tiene lugar bajo la zona fundida.

- La influencia de las interacciones térmicas y mecánicas en la interfase del sustrato-recubrimiento influyen significativamente en el desarrollo de la adherencia de los mismos durante la proyección térmica. Las interacciones térmicas entre el sustrato y el recubrimiento conducen a una fusión parcial o completa de la región interfacial del sustrato, y esta región fundida puede enlazarse con el recubrimiento. En el caso de superficie de sustrato rugosa, el tamaño de esta zona fundida es mayor que no para la superficie pulida. Como resultado, la rugosidad del sustrato permite un mayor tiempo para desarrollar una buena adherencia recubrimiento-sustrato.

- Los resultados teóricos concuerdan con los datos experimentales.

\section{Agradecimientos}

Los autores quieren agradecer a la Generalitat de Catalunya (proyecto GRQ93-1017) y a la CICYT (proyecto MAT96-0426) el soporte financiero recibido. El prof. V.V. Sobolev agradece a la Generalitat de Catalunya la concesión de una ayuda como Profesor Visitante en la U.B. J.A. Calero también agradece a la Generalitat de Catalunya la concesión de una beca F.I.

\section{REFERENCIAS}

(1) Berndt, C.C., Brindley, W., Goland, A.N., Herman, H., Houck, D.L., Jones, K., Miller, R.A., Neiser, R, Riggs, W., Sampath, S, Smith, M y Spanne, P. J. Therm. Spray Technol., 1 (4), 1992: 341-356.

(2) Sobolev, V.V., Gullemany, J.M. y Calero, J.A. Mater. Sci. Technol., 11 (8), 1995: 810-819.

(3) Sobolev, V.V., Guilemany, J.M. y Calero, J.A. Mater. Mater. Sci. Technol., 11 (10), 1995: 1.052-1.059.

(4) Sobolev, V.V., Gullemany, J.M., Miguel, J.R. y CaleRO, J.A. Surf. Coat. Technol., 82, 1996: 121-129.

(5) Sobolev, V.V., Guilemany, J.M., Miguel, J.R. y CaleRO, J.A. Surf. Coat. Technol., 82, 1996: 114-120.

(6) Sobolev, V.V. y Guilemany, J.M. Mater. Lett., 28, 1996: 331-335.

(7) Sobolev, V.V., Guilemany, J.M. Calero, J.A. y Villuendas, F.J. J. Therm. Spray Technol., 4 (4), 1995: 408-414.

(8) Sobolev, V.V., Gullemany, J.M. y Calero, J.A. $J$. Therm. Spray Technol., 4 (4), 1995: 401-407.

(9) Sobolev, V.V., Guilemany, J.M. y Martin, A.J. $J$. Therm. Spray Technol., 5 (2), 1996: 207.214

(10) Sobolev, V.V. y Gullemany, J.M. Int. Mater. Rev., 41 (1), 1996: 13-32.

(11) Sobolev, V.V. y Gullemany, J.M. Mater. Lett., 28, 1996: 71-75.

(12) Sobolev, V.V., Gullemany, J.M. y Calero, J.A. $J$. Therm. Spray Technol., en prensa, 1996.

(13) Sobolev V.V. y Guilemany, J.M. Mater. Lett., 18, 1994: 304-308.

(14) Sobolev V.V. y Gullemany, J.M. Surf. Coat. Technol., 70, 1994: 57-68.

(15) Sobolev, V.V. y Guilemany, J.M. J. Mater. Process. Technol., 58, 1996: 227-232.

(16) Gullemany, J.M., Sobolev, V.V., Nutting, J., Dong, Z. y CALERo, J.A. Scr. Metall. Mater., 31 (7), 1994: 915-920.

(17) Nutting, J., Gullemany, J.M. y Dong, Z. Mater. Sci. Technol., 11 (9), 1995: 961-966.

(18) Guilemany, J.M., Nutting, J., Dong, Z. y de Paco, J.M. Scr. Metall. Mater., 33 (7), 1995: 1.055-1.062.

(19) Gullemany, J.M., Nutting, J. y Dong, Z. Mater. Res. Bull., pendiente de publicar, 1996.

(20) Luborsky, F.E. De., Amorphous Metallic Alloys. Ed. Butterworths, Londres, 1983: 144-168.

(21) Kurz, W. y Fisher, D.J. Fundamentals of Solidification. Ed. Trans. Techn., Aedermannsdorf (Suiza), 1984: 65-97.

(22) Moreau, C., Gougeon, P. y Lamontagne, M. J. Therm. Spray Technol., 4 (1), 1995: 25-33. 